\title{
Broadband data aided velocity model building
} and imaging

\author{
D. Vigh* (Schlumberger), N. Moldoveanu (Schlumberger), J. Kapoor \\ (Schlumberger), K. Jiao (Schlumberger) \& G. Menzel-Jones (Schlumberger)
}

\section{SUMMARY}

During the past decade, marine seismic acquisition evolved from narrow-azimuth (NAZ) to wide-azimuth (WAZ) geometries toward the latest offering of long-offset full-azimuth (FAZ) surveys. This advance in acquisition design is mainly driven by the demands of improved illumination in complex areas such as Gulf of Mexico (GOM) subsalt environments. Despite these acquisition and processing improvements, marine data still suffers from the ghost effect of free-surface reflections and resulting in constructive and destructive interference between the up- and down-going wavefields. If uncorrected, the ghost significantly reduces the bandwidth and distorts the phase of the recorded data and prevents us from producing a broadband image. Fortunately, the ghost effect is straightforward to simulate and, therefore, to correct, either prior to or during two-way wave equation inversion and imaging. Optimal use of this improved data, two-way wave equation algorithms such as reverse time migration (RTM) and full waveform inversion (FWI) are required. Their high-fidelity nature enables them to honor complex velocity fields with the accuracy required, particularly for migrating long-offset data, and as a result, for producing satisfactory model refinements and images when other methods fail. Finally, even with the best acquisition and imaging algorithms, RTM images can be further improved by post-imaging enhancements using vector image partitions (VIPs).

We demonstrate an efficient workflow to maximize the power of broadband acquisition by applying velocity model building with FWI, imaging with RTM compensation for ghost effects in both steps, and outputting partial images for further optimization. 


\section{Introduction}

Wide-azimuth (WAZ) towed-streamer acquisition has proven to be a successful technique for subsalt imaging in the Gulf of Mexico. Numerous survey and imaging projects have been undertaken to achieve quality subsalt images. However, the rapid development in imaging and modeling algorithms demand a more advanced acquisition technique that offers even better signal-to-noise ratio, illumination, and azimuth information. One of the viable approaches that offer an even distribution of azimuth information is the circular shooting geometry with slanted cable. These recent advances in acquiring data, such as the dual coil method, offer significant improvements in characteristics over WAZ and ocean-bottom cable such as better illumination, lower frequencies, and longer offsets, up to and beyond $14 \mathrm{~km}$. These advancements allow full waveform inversion (FWI) to more accurately and deeper determine the velocity fields that are key to the imaging algorithms. Usually, the anisotropic fields are used in data simulation or forward propagation but they are not updated during the FWI iterations that require a multiparameter FWI approach. In the past few years there have been several suggestions on how to approach multiparameter FWI. Because data acquisition is progressing toward longer and longer offsets, anisotropy is becoming a pressing issue, inverting for at least the anisotropic parameters. However, inverting for a increased number of parameters further complicate the already non-linear inversion approach ( Plessix, 2011). Considering the importance of data with long offsets and low frequencies, in most geologic environments, anisotropy is an unavoidable topic for FWI. The results demonstrate that FWI can be used for velocity updates with long offsets and low frequencies provided by the above described dual-coil seismic acquisition with slanted cables on the receiver side.

\section{Field data description and results}

The data set is a result of dual-coil acquisition where the maximum offset is up to $14,000 \mathrm{~m}$ with fullazimuth distribution. The shooting is circular with ten towed cables on each recording vessel; the cables were slanted with variable depths up to a $40 \mathrm{~m}$ maximum depth to achieve broadband data collection (Kroode, 2013). The output size exceeds $450 \mathrm{~km} 2$ with approximately 104,000 shots covering the part of Alaminios Canyon are of Gulf of Mexico (Figure 1a. and $1 \mathrm{~b}$.) using the dual-coil layout mentioned above. The gun array, the shot depth, and the cable depth allowed us to observe low frequencies of about 2.5 to $3 \mathrm{~Hz}$ on the field records. A $50-\mathrm{m}$ by $60-\mathrm{m}$ bin size was selected to run the inversion. The inversion utilized anisotropic propagators with tilted transverse isotropy, and the inversion updates velocity and epsilon while $\delta$ are derived from well information and extrapolated to the target area. $\theta$ and $\phi$ are derived from a vintage vertical transverse isotropic reverse time migrated image. The starting velocity was a long-scale-length ray-based tomography velocity. The subsalt velocity and anisotropic fields were built by superposing a regional velocity trend based upon existing wells below the salt. The FWI at the starting frequency $(2.8 \mathrm{~Hz})$ updated the velocity field first because it has the first-order impact on the kinematics versus the epsilon update .After reducing the misfit energy at $2.8 \mathrm{~Hz}$ the velocity update was continued at the next frequency $(3.5 \mathrm{~Hz})$ which produced further misfit only reduction relying on the velocity update. Our sequential strategy included an epsilon inversion using the observed data at $3.0 \mathrm{~Hz}$ following the two velocity updates. Even though our offset was up to $14 \mathrm{~km}$, this behaviour can be explained by the fact that the anisotropy has second-order impact on the kinematics. In spite of the second-order effect of epsilon on the kinematics it can further flatten the gathers and derive a correct anisotropic velocity volume taking the imaging condition described above into account. The starting parameters are very smooth and simple, while, after the updates on both, velocity and epsilon conform to the geology when the depth slices are considered. The vertical section demonstrates a tremendous uplift in imaging after application of the multiparameter FWI. Because the acquisition was broadband the reverse time migration included the deghosting on the source and the receiver side too (Figure 1c.and 1d.). 

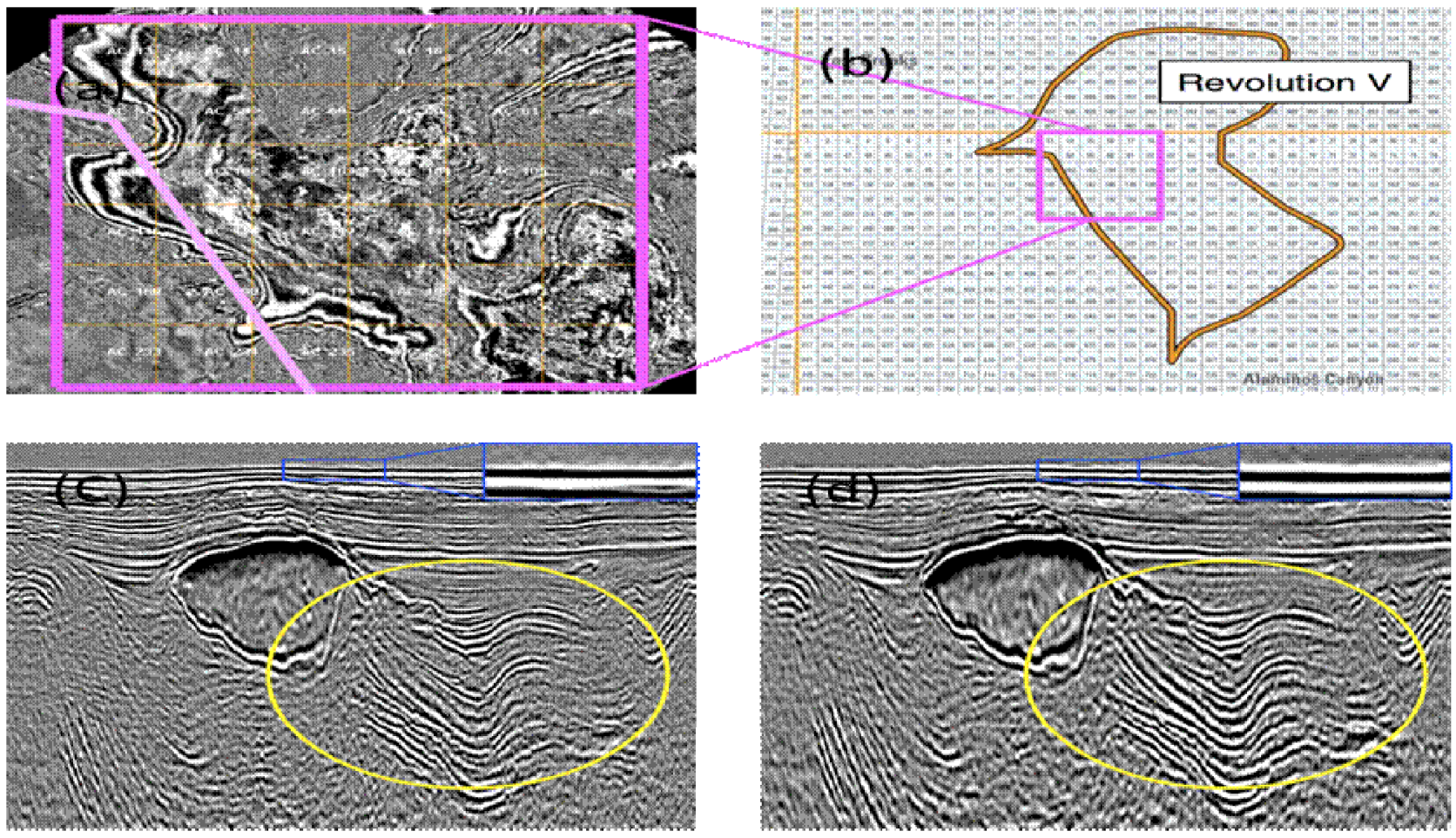

Figure 1 Location map and imaging. (a) Zoomed in location map (b) Area in term of Gulf of Mexico

(c) Traditional reverse time migration (d) Ghosted reverse time migration

\section{Conclusions}

We demonstrated a successful strategy and application of multiparameter FWI that produced geologically plausible velocity and epsilon fields to improve broadband long-offset imaging in the Gulf of Mexico. When long offsets are used in acquisition, the anisotropic imaging condition should be employed rather than only including its parameters in the propagators, which ensures that the velocity and epsilon better represent the Earth parameters.

\section{References:}

Kroode, F., Bergler, S., Corsten C., Willem de Magg, J., Stijbos, F. and Tijhof, H. [2013] Broadband seismic data - The importance of low freqiemcies, Geophysics, VOL 78.NO 2, P.WA 3 WA 14.

Plessix, R.E. and Cao, Q. [2011] A parametrization study for surface seismic full waveform inversion in an acoustic vertical transversely isotropic medium. Geophysical Journal International, 1 85(1), 539-556. 